\title{
A Second-Order-Based Decision Tool for Evaluating Decisions Under Conditions of Severe Uncertainty
}

\author{
Mats Danielson ${ }^{1,2}$, Love Ekenberg ${ }^{2,1}$, and Aron Larsson ${ }^{1,3}$ \\ ${ }^{1}$ Dept. of Computer and Systems Sciences, Stockholm University \\ PO Box 7003, SE-164 07 Kista, Sweden; mats.danielson@su.se \\ ${ }^{2}$ International Institute for Applied Systems Analysis, IIASA \\ Schlossplatz 1, A-2361 Laxenburg, Austria; ekenberg@iiasa.ac.at \\ ${ }^{3}$ Dept. of Information Systems and Technology, Mid Sweden University \\ SE-851 70 Sundsvall, Sweden; aron@dsv.su.se
}

\begin{abstract}
The requirement to assign precise numerical values to model entities such as criteria weights, probabilities, and utilities is too strong in most real-life decision situations, and hence alternative representations and evaluation mechanisms are important to consider. In this paper, we discuss the DecideIT 3.0 state-of-the-art software decision tool and demonstrate its functionality using a real-life case. The tool is based on a belief mass interpretation of the decision information, where the components are imprecise by means of intervals and qualitative estimates, and we discuss how multiplicative and additive aggregations influence the resulting distribution over the expected values.
\end{abstract}

Keywords: Decision analysis, decision software, imprecise criteria weights, imprecise probabilities.

\section{Introduction}

There have been many suggestions for how to deal with the strong requirements of most decision models to provide precise information. One basic idea is to simply assign homogenous distributions over the variables, but this is done to the cost of significant information loss, which is why it is preferred to at least ordinally or cardinally rank the components such as probabilities, utilities, and criteria weights, and thereafter utilise one of several techniques to handle them. A quite useful approach is to utilise surrogate weights, derived from the rankings, and several authors have suggested various means for that, such as (Stewart, 1993; Arbel and Vargas, 1993; Barron and Barrett, 1996a,b; Katsikopoulos and Fasolo, 2006), and many others. The rankings are then transformed into numerical weights by dedicated mapping functions. Despite some controversies, many surrogate weight methods have been suggested, such as in (Stillwell et al., 1981) rank-sum weights and rank reciprocal weights), ROC (rank order centroid), e.g., Barron (1992). Entropy arguments also occur, such as (Ahn, 2011; Barron, 1988; Barton and Barrett, 1996b; Jaynes, 1968). Yager (1988) used an ordered weighting average method and (Fuller and Majlender, 2001, 2003) used the Maximum Entropy OWA (MEOWA) method based on minimal variability. The MEOWA is, however, a more complex 
measure than, e.g., ROC, and requires an attitude (similar to a pessimism-optimism) parameter. In many situations, there is a need utilise entirely different frameworks for representing vagueness, such as the theory of capacities, sets of probability measures, interval probabilities, evidence and possibility theories, fuzzy measures, preference rankings, and higher-order probability theory, or combinations of rankings with other representation formats (see for example Dubois, 2010; Rohmer and Baudrit, 2010; Shapiro and Koissi, 2015; Danielson et al., 2014; Dutta, 2018; Herrera and HerreraViedma, 2000; Jansen et al., 2018 to name just a few in the extensive literature in the fields). Often, for these theories to be reasonably transparent to the decision-maker, (s)he is required to possess significant mathematical knowledge, and even then sometimes the theories include relatively harsh (and hence non-transparent) methods for discriminating between decision alternatives. Furthermore, the computational complexity can be high in various respects, as we have argued (for an extensive background, see, e.g., (Danielson and Ekenberg, 2007)), and there is a strong need for user-friendly tool support, while still maintaining a high capacity for evaluations of a wide range of assessment types.

We have during the last 20 years created evaluation software for these purposes. Our earlier versions of decision support software have been successfully used in a wide variety of contexts, e.g., long-term storage of nuclear waste, land use planning, choice of insurance portfolios against catastrophe events, massive-scale energy policy formation, gold mining evaluations, health-care planning, assessments for medical risks, emergency management, and so on (Fasth et al., 2020; Hansson et al., 2011; Komendantova et al., 2018; Mihai et al., 2015; Kivinuke et al., 2015; Caster et al., 2012; Larsson et al., 2010).

In this paper, we demonstrate a significantly extended software tool DecideIT 3.0 (Preference AB, 2019), implementing our latest findings regarding aggregations of distributions. This is a landmark in the handling of imprecise information and differs significantly from earlier versions of the tool which handled only a pure interval approach with orderings. In the earlier versions, the result of an evaluation still contained unnecessary uncertainty regarding the final outcome. This is due to overlaps in expected values making it difficult to discriminate between the alternative options involved. By adding second-order information in the way done in DecideIT 3.0, we can significantly enhance a decision-maker's understanding of decision situations when handling aggregations of imprecise representations.

We will thus in this paper discuss the underlying framework enabling evaluations that are subject to incomplete input data. The software is able to evaluate decision situations including imprecise utilities, probabilities, and criteria weights, and qualitative estimates between these components. We avoid the introduction of new concepts into the decision models, such as set membership functions or similar formalisms, and instead use higher-order distributions of belief in the basic utilities, probabilities, and criteria weights which then allows for better and more transparent discrimination between the resulting values of the decision alternatives. The ability to use ordinary belief distributions over probabilities, values, and criteria weights enhances the transparency of the results since no new concepts have to be introduced in the evaluation of the model. There are no other known software tools (including earlier versions of DecideIT) that are able to calculate the resulting belief in expected 
values based on user input in terms of belief in probabilities, values, and criteria weights.

The next sections cover the decision-theoretical framework and explain the data model, and are followed by a description of an application to a real-life problem involving the largest Swedish energy provider. The presentation starts with the representation model in which the decision data is structured and stored. The tool supports elicitation models as discussed in (Riabacke et al., 2012). Having successfully elicited and stored the decision data in the tool, the evaluation can commence. Thus, the evaluation model is described next. To illuminate the entire process, a real-life example concludes the paper, in which the framework and the tool are utilised. Needless to say, in a real-life situation, the decision-maker(s) will iterate between the elicitation and evaluation steps, and also iterate repeatedly within the steps.

\section{Representation Model}

Probabilistic decision situations are often described by a decision tree, as shown in Figure 1.

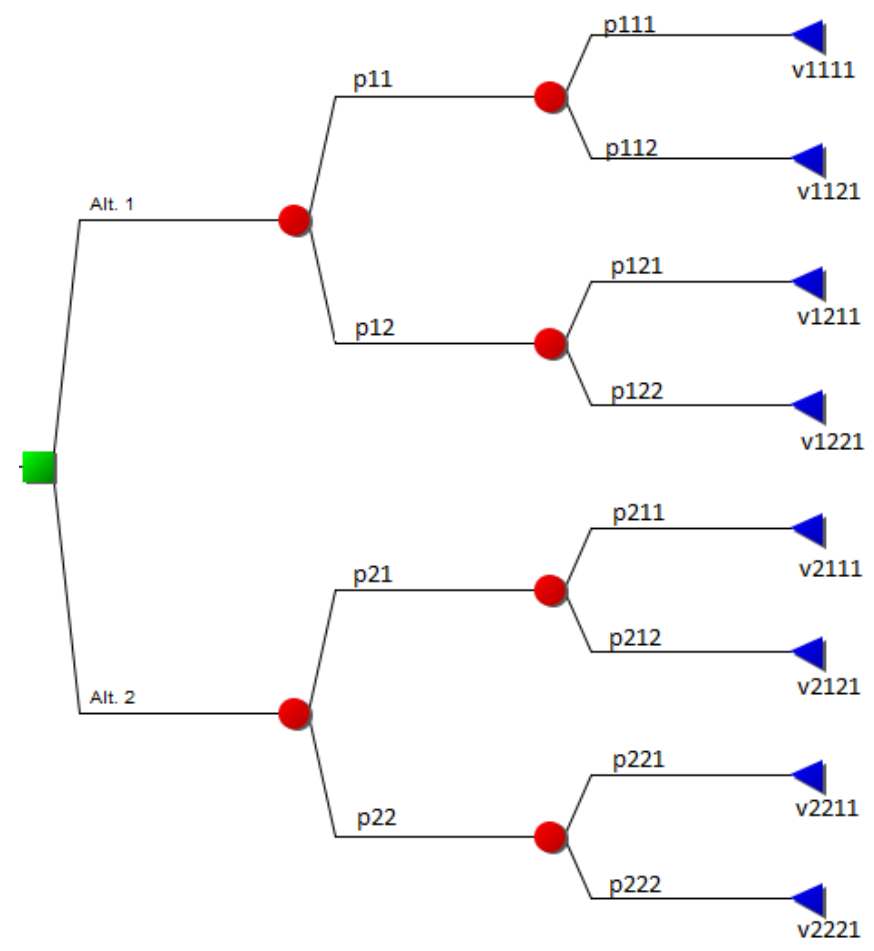

Figure 1 A decision tree representation of a probabilistic decision situation

The components of such a decision tree are a root node (also called a decision node), a set of probability nodes (representing uncertainty) and consequence nodes (the final outcomes). The probability and consequence nodes are in a standard model assigned 
unique numerical probability and value distributions. The semantics employed here are as follows: when an alternative $A_{i}$ is selected, there is a probability $\mathrm{p}_{\mathrm{ij}}$ that an event occurs that leads either to another subsequent event or to a consequence with a value $\mathrm{v}_{\mathrm{ijk}}$. A common evaluation rule in this context is the maximisation of the expected value; for instance, in the case of alternative $A_{i}$ in Figure 1, the expected value is:

$$
E\left(A_{i}\right)=\sum_{j=1}^{2} p_{i j} \sum_{k=1}^{2} p_{i j k} v_{i j k 1},
$$

which can be straightforwardly generalised to the multi-linear equation:

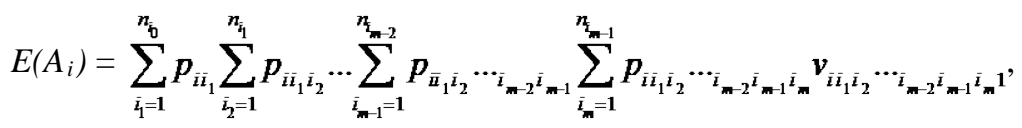

where the $p_{i j}$ with varying indices denote probabilities and the $v_{i j}$ counterparts denote values.

This modelling can be generalised to cases where there is imprecise or incomplete information with respect to probabilities and consequence or alternative values, which we collect using the concept of a multi-frame to gather all necessary information. We discuss the theory for this below in an informal way.

User statements may be range constraints or comparative statements, which are translated into systems of inequalities in a value constraint set. Probability statements are collected in a node constraint set. User statements have the following forms:

- Range constraints: a probability or value $\mathrm{y}_{\mathrm{i}}$ lies between $\mathrm{a}_{1}$ and $\mathrm{a}_{2}$, denoted as $\mathrm{y}_{\mathrm{i}} \in$ $\left[\mathrm{a}_{1}, \mathrm{a}_{2}\right]$ and represented by $\mathrm{y}_{\mathrm{i}}>\mathrm{a}_{1}$ and $\mathrm{y}_{\mathrm{i}}<\mathrm{a}_{2}$ for real numbers $\mathrm{a}_{1}$ and $\mathrm{a}_{2}$.

- Comparisons: $\mathrm{y}_{\mathrm{i}}$ is larger than $\mathrm{y}_{\mathrm{j}}$ by a difference from $\mathrm{d}_{1}$ to $\mathrm{d}_{2}$, denoted $\mathrm{y}_{\mathrm{i}}-\mathrm{y}_{\mathrm{j}} \in$ $\left[\mathrm{d}_{1}, \mathrm{~d}_{2}\right]$ and represented by $\mathrm{y}_{\mathrm{i}}-\mathrm{y}_{\mathrm{j}}>\mathrm{d}_{1}$ and $\mathrm{y}_{\mathrm{i}}-\mathrm{y}_{\mathrm{j}}<\mathrm{d}_{2}$, for real numbers $\mathrm{d}_{1}$ and $\mathrm{d}_{2}$.

Constraint sets thus consist of linear inequalities and probability and value node constraint sets, characterising sets of (discrete) probabilities and distributions. Probability node constraint sets also involve the usual normalisation constraints $\left(\sum_{\mathrm{j}} \mathrm{X}_{\mathrm{ij}}=1\right)$ that require the probabilities to sum to one.

Let $\mathrm{T}$ be a consequence tree and $\mathrm{N}$ be a constraint set for the variables $\left\{\mathrm{n}_{\ldots} \ldots \mathrm{i} \ldots \mathrm{j} \ldots\right\}$.

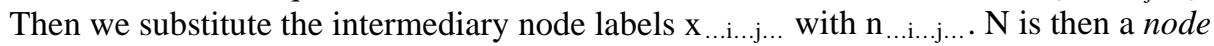
constraint set for $\mathrm{T}$ if for all sets $\left\{\mathrm{n}_{\ldots \mathrm{i}}, \ldots, \mathrm{n}_{\ldots} . . \mathrm{im}\right\}$ of all sub-nodes of nodes $\mathrm{n}_{\ldots . \mathrm{i}}$ that are not leaves, the statements $\mathrm{n}_{\ldots \mathrm{ij}} \in[0,1]$ and $\sum_{\mathrm{j}} \mathrm{n}_{\ldots \mathrm{ij}}=1, \mathrm{j} \in[1, \ldots, \mathrm{m}]$ are in N. We will also use the term multi-frame as a structure $\langle\mathrm{T}, \mathrm{N}\rangle$, where $\mathrm{T}$ is a consequence tree and $\mathrm{N}$ is a set of all constraints sets relative to $\mathrm{T}$.

Given a set of variables $\left\{\mathrm{x}_{\mathrm{i}}\right\}_{\mathrm{i} \in \mathrm{I}}$ a solution to a system $\mathrm{X}$ of inequalities in $\left\{\mathrm{x}_{\mathrm{i}}\right\}_{\mathrm{i} \in \mathrm{I}}$ is a real vector $\mathbf{a}=\left(a_{1}, \ldots, a_{n}\right)$ where each $a_{i}$ is substituted for $x_{i}$ such that every inequality in the system is satisfied. (There exists a solution if the substitution of $a_{i}$ for $x_{i}$ in $X$, for all $1 \leq \mathrm{i} \leq \mathrm{n}$, does not yield a contradiction). The vector $\mathbf{a}$ is called a solution vector to $X$. The solution set for $X$ is $\{\mathbf{b} \mid \mathbf{b}$ is a solution to $X\}$. A constraint set $X$ in $\left\{x_{i}\right\}_{i \in I}$ is consistent iff the system of weak inequalities in $\mathrm{X}$ has a solution. A minimal requirement is that there must exist some vector of variable assignments that simultaneously satisfies each inequality in the constraint sets, i.e., given a consistent constraint set $\mathrm{X}$ in the variables $\left\{\mathrm{x}_{\mathrm{i}}\right\}, \mathrm{X}_{\max }\left(\mathrm{x}_{\mathrm{i}}\right)={ }_{\text {def }} \sup \left(\mathrm{a} \mid\left\{\mathrm{x}_{\mathrm{i}}>\mathrm{a}\right\} \cup \mathrm{X}\right.$ is consistent $)$. 
Similarly, $\mathrm{x}_{\min }\left(\mathrm{x}_{\mathrm{i}}\right)={ }_{\text {def }} \inf \left(\mathrm{a} \mid\left\{\mathrm{x}_{\mathrm{i}}<\mathrm{a}\right\} \cup \mathrm{X}\right.$ is consistent). Furthermore, given a function $f, x_{\operatorname{argmax}}(f(x))$ is a solution vector that is a solution to $x_{\max }(f(x))$, and $x_{\operatorname{argmin}}(f(x))$ is a solution vector that is a solution to $x_{\min }(f(x))$. Finally, the set of orthogonal projections of the solution set is the orthogonal hull, more formally defined as the set of pairs $\left\langle\mathrm{x}_{\min }\left(\mathrm{x}_{\mathrm{i}}\right), \mathrm{X}_{\max }\left(\mathrm{x}_{\mathrm{i}}\right)\right\rangle$ given a consistent constraint set $\mathrm{X}$ in $\left\{\mathrm{x}_{\mathrm{i}}\right\}_{\mathrm{i} \in[1, \ldots \mathrm{n}]}$.

An orthogonal hull is thus straightforwardly found by solving a set of linear programming problems in standard fashion.

\section{Introducing Second-Order Beliefs}

When specifying an interval, the actual beliefs in the values are probably not uniformly distributed. Earlier versions of the DecideIT tool handled such second-order belief approaches only to a limited extent in essentially two different ways, by contraction analysis and by Monte Carlo simulations. Contraction analysis was done by decreasing the interval widths by "contracting” the interval endpoints towards a focal point, the latter either being provided by the decision-maker or suggested as the centre of mass of the polytope defined by the intervals. Hence, the second-order representation was not explicit as the contraction analysis relied on the assumption that points closer to the centre of mass have larger belief mass compared to points closer to extreme points of the intervals. The amount of contraction (percentage of intervals cut off from the ends) required until the expression $\min \left[E\left(A_{i}\right)-E\left(A_{j}\right)\right]<0$ was not satisfied in any single point of the remaining polytope (i.e. there existed no solution) was therefore viewed as a measure of robustness for a preference in favour of an alternative $A_{i}$ compared to another alternative $A_{j}$. The level of contraction was given as a percentage of the original intervals but was restricted with respect to the granularity of the analyses. See, (Danielson et al., 2007) for an extended presentation.

The Monte Carlo simulation approach took advantage of transformations between randomly generated points in a unit cube and a subset of the cube constructed from a user stipulated constraint set. A sampling algorithm was used for generating Dirichlet distributed probabilities (Tervonen and Lahdelma, 2007) while a factorization of a joint uniform distribution was used for generating ordered utility variables in the case of comparative constraints. See (Larsson et al., 2014) for an account of this approach. The simulation approach could not allow both for upper probability bounds and for partial rankings of consequences and probabilities.

As a significant improvement over these earlier attempts, the tool now utilises belief distributions that indicate the strengths with which a decision-maker believes in these different values, and this approach is able to evaluate the results without any of the former constraints. The key differences can be seen in Table 1.

\begin{tabular}{|l|c|c|c|}
\hline Functionality $\quad$ Version & $\mathbf{1 . 0}$ & $\mathbf{2 . 0}$ & $\mathbf{3 . 0}$ \\
\hline Probabilistic models & $\bullet$ & $\bullet$ & $\bullet$ \\
\hline Probability intervals & $\bullet$ & $\bullet$ & $\bullet$ \\
\hline Utility/value intervals & $\bullet$ & $\bullet$ & $\bullet$ \\
\hline Multi-criteria models & & $\bullet$ & $\bullet$ \\
\hline Combined prob. and multi-criteria & & $\bullet$ & $\bullet$ \\
\hline
\end{tabular}




\begin{tabular}{|l|c|c|c|}
\hline $\begin{array}{l}\text { Evaluation using contractions } \\
\text { (first-order information) }\end{array}$ & $\bullet$ & $\bullet$ & $(\bullet)$ \\
\hline $\begin{array}{l}\text { Evaluation using belief mass } \\
\text { (second-order information) }\end{array}$ & & & $\bullet$ \\
\hline
\end{tabular}

Table 1. Key DecideIT functionality

In this extended model, we first introduce parameters for belief distributions for probabilities and values; thereafter, we can operate on these distributions by utilising additive and multiplicative combination rules for random variables. ${ }^{1}$

A unit cube is all tuples $\left(x_{1}, \ldots, x_{\mathrm{n}}\right)$ in $[0,1]^{n}$ and a second-order distribution over such a cube $B$ is a positive distribution $F$ defined on $B$ such that

$$
\int_{B} F(x) d V_{B}(x)=1 \text {, where } V_{\mathrm{B}} \text { is the n-dimensional Lebesgue measure on } B \text {. }
$$

We use different distributions for probabilities and values because of the normalisation constraints for probabilities; natural candidates are the Dirichlet distribution for probabilities and two- or three-point distributions for values. The properties of the Dirichlet distribution as a parameterised family of continuous multivariate probability distributions make it suitable for this purpose.

The probability density function of the Dirichlet distribution is defined as

$$
f_{\text {dir }}(p, \alpha)=\frac{\Gamma\left(\sum_{i=1}^{k} \alpha_{i}\right)}{\prod_{i=1}^{k} \Gamma\left(\alpha_{i}\right)} p_{1}^{\alpha_{i}-1} p_{2}^{\alpha_{2}-1} \ldots p_{k}{ }^{\alpha_{k}-1}
$$

on a set $\left\{\mathrm{p}=\left(\mathrm{p}_{1}, \ldots \mathrm{p}_{\mathrm{k}}\right) \mid \mathrm{p}_{1}, \ldots, \mathrm{p}_{\mathrm{k}} \geq 0, \Sigma \mathrm{p}_{\mathrm{i}}=1\right\}$, where $\left(\alpha_{1}, \ldots, \alpha_{\mathrm{k}}\right)$ is a parameter vector in which each $\alpha_{\mathrm{i}}>0$ and $\Gamma\left(\alpha_{\mathrm{i}}\right)$ is the Gamma function. ${ }^{2}$

The Dirichlet distribution is thus a multivariate generalisation of the beta distribution, and the marginal distributions of Dirichlet are beta distributions. For instance, when a distribution is uniform, the marginal distribution is a polynomial of degree $n-2$, where $\mathrm{n}$ is the dimension of a cube $\mathrm{B}$, i.e. when all $\alpha_{\mathrm{i}}=1$, then the Dirichlet distribution is uniform with the marginal distribution

$$
f\left(x_{i}\right)=\int_{B_{i}^{-}} d V_{B_{i}^{-}}(x)=(n-1)\left(1-x_{i}\right)^{n-2}
$$

For our purposes, we use a different form, namely the bounded Dirichlet distribution over a (normally user-specified) range instead of the interval $[0,1]$. Bounded beta

1 The detailed background theory of belief distributions and aggregations in this sense is described in (Ekenberg and Thorbiörnson, 2001; Ekenberg et al., 2006; Danielson et al., 2007, Ekenberg et al., 2014; Sundgren et al., 2009).

2 The details of this are provided in any standard textbook on Bayesian statistics, such as (Kendall and Stewart, 1969). 
distributions are then derived from this, giving four-parameter beta distributions. Thus, we define a probability belief distribution through a bounded Dirichlet distribution $f_{3}\left(a_{i}, c_{i}, b_{i}\right)$ where $c_{i}$ is the estimated most likely probability and where $a_{i}$ and $b_{i}$ are the boundaries for the support of the distribution $\left(a_{i}<c_{i}<b_{i}\right)$ (cf. Kotz and van Dorp, 2004).

For the values (i.e. without the normalisation constraint), the generalisation to a trapezoid is straightforward. A delta distribution is a two-point distribution (uniform or trapezoidal) or a three-point distribution (triangular). When we have no reason to make any other specific assumptions, for instance when there is large uncertainty in the underlying belief distributions involved, a two-point distribution modelling the upper and lower bounds (the uniform or trapezoid distributions) seems to be reasonable, even if this is seldom the case. However, when modal outcomes can be estimated to some extent, the beliefs would probably be better represented by three-point distributions. In this case, Beta and Erlang belief distributions generally give results similar to triangular distributions. Here, we assume that we only have limited sample data, but that the variable relationships are known in addition to the minima, maxima, and modal values. For instance, Golenko-Ginzburg (1988) discusses PERT networks and their distributions. The mean value of a number of three-point value belief distributions $f_{3}\left(a_{i}, c_{i}, b_{i}\right)$ is $\mu(\lambda)=\left(a_{i}+b_{i}+\lambda c_{i}\right) /(\lambda+2)$, with $\lambda=1$ for triangular distributions and $\lambda=0$ for a two-point uniform or trapezoid distribution. ${ }^{3}$ For practical purposes, there is normally no reason to use any three-point distribution other than a triangular distribution, since the risk of underestimation is lower. When the decision data has been successfully elicited, the evaluation model can be applied to the data. This is covered in a later section, while the next section provides a brief review of our previous approaches to deal with second-order beliefs in the DecideIT software.

\section{Evaluation Model}

The evaluation model is based on the resulting distribution over the generalised expected utility mentioned above, i.e.,

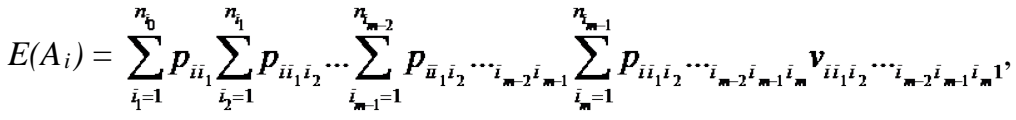

where we have distributions over the random variables $p$ and $v$. Let $G$ be a distribution over two cubes $A$ and $B$, and assume that $G$ has positive support for the feasible distributions at level $i$ in a decision tree, and for the feasible probability distributions of the children of a node $x_{i j}$. Furthermore, assume that $f(x)$ and $g(y)$ are marginal distributions of $G(z)$ on $A$ and $B$, respectively. Then the cumulative multiplied distribution of the two belief distributions is

$$
\mathrm{H}(\mathrm{z})=\iint_{\Gamma_{z}} f(x) g(y) d x d y=\int_{0}^{1} \int_{0}^{z / x} f(x) g(y) d x d y=\int_{z}^{1} f(x) G(z / x) d x
$$

${ }^{3}$ Beta-PERT usually uses $\lambda=4$ and Erlang-PERT $\lambda=3$. However, higher values of $\lambda$ tend to underestimate the uncertainties involved. 
where $\mathrm{G}$ is a primitive function of $\mathrm{g}, \Gamma_{z}=\{(x, y) \mid x \cdot y \leq z\}$, and $0 \leq z \leq 1$.

Let $h(z)$ be the corresponding density function. Then

$$
\mathrm{h}(\mathrm{z})=\frac{d}{d z} \int_{z}^{1} f(x) G(z / x) d x=\int_{z}^{1} \frac{f(x) g(z / x)}{x} d x .
$$

Addition is handled by the standard convolution of two densities, restricted to A and B and the distribution h on a sum $z=x+y$, where we have the belief distributions $f(x)$ and $g(y)$ is consequently given by

$$
\mathrm{h}(\mathrm{z})=\frac{d}{d z} \int_{0}^{z} f(x) g(z-x) d x .
$$

Iterating this, we obtain the distribution over the generalised expected utility.

\subsection{The B-normal Method}

The B-normal (business normal) method employs distributions that are qualified by assumptions made about the environment in which the method is going to be applied. These assumptions involve the frequency of the decisions made and the survival of the business over time. They allow methods from both risk and decision theory and business administration to be employed in forming the B-normal decision method.

In the same way as in standard risk and decision theory, we assume that a large number of events will occur and a large number of decisions will be made. In this way, maximising the expected value becomes a reasonable decision rule, and at the same time, the belief distributions over the expected values tend to normal distributions or distributions of a similar kind. Note that even when we assume that the expectations are estimated a large number of times (due to repeated decision making) and can consequently be approximated by a normal distribution, there are three observations in particular that should be considered here:

1. The resulting distributions will be approximately normal only when the original distributions are symmetric, which of course is not usually the case for beta and triangular distributions. The result will instead be skew-normal.

2. Even if the original distributions are symmetric, the non-linear multiplication operator breaks the symmetry. The result will again be approximately skewnormal.

3. To obtain a resulting approximate normal distribution, both the original distributions and their aggregations must allow for long tails. In general, this is not the case in our situation; this is because our estimates have lower and upper limits due to the fact that we use bounded Dirichlet distributions and uniform and triangular distributions, yielding approximately truncated normal distributions.

We therefore employ the skew-normal distribution to generalise the normal distribution by allowing for non-zero skewness, i.e. asymmetry. This is accomplished by introducing a shape parameter $\alpha$, where $\alpha=0$ represents the standard normal distribution, and $\alpha=1$ yields the distribution of the maximum of two independent 
standard normal variates. We can then conveniently represent truncated (skew-)normal distributions as probability distributions of (skew-)normally distributed random variables that are bounded. The skewness of the distribution increases with the absolute value of $\alpha$, and when $|\alpha| \rightarrow \infty$, we get folded normal or half-normal distributions. Distributions are right-skewed when $\alpha>0$ and left-skewed when $\alpha<0$. When the sign of $\alpha$ is changed, the density is reflected about $x=0$. The skew-normal probability density function with location $\xi$, scale $\omega$, and shape parameter $\alpha$ is

$$
f(x)=\frac{2}{\omega} \varphi\left(\frac{x-\xi}{\omega}\right) \Phi\left(\alpha\left(\frac{x-\xi}{\omega}\right)\right)
$$

which turns into a standard normal distribution for $\alpha=0$.

Assume that a distribution $X$ has a normal distribution within the interval (a, b). Then $X, a<X<b$, has a truncated normal distribution and its probability density function is given by a four-parameter expression that tends to normality as the intervals are widened (see for instance Loeve, 1977).

The B-normal distribution method employs a skew-normal distribution as above, adapting it to business use. It expresses the resulting distribution of outcomes of events in the context of business operations. The joint distribution of all modelled consequence pairs is approximately skew-normally distributed, and this approximation improves as the number of consequence pairs increases.

The adaptations of skew-normality to business use (B-normality) consist of:

- location and scale parameters that match the expected value and variance with the normal distribution while maintaining the same skewness;

- $\quad$ interpolated truncation toward the theoretical minimum and maximum expected values (i.e. the approximation tails extend further);

- $\quad$ handling of large skew, where standard skew-normality does not hold, by a successive limiting of the shape parameter;

- $\quad$ interpretation of binary risk trees as a going concern (the business operates over an indefinite time in the future).

To employ the B-normal method, the skewed distribution must be aligned to give the same variance and expected value as its unskewed counterpart and must display the correct shape (skew). Assuming that the desired expected value is $E(X)$, the desired variance is $\sigma^{2}$, and the desired skew is $s$. The alignment (matching) of the B-normal distribution is done in three steps:

1. Obtain the shape parameter $\alpha$ that describes the desired skew $s$ of a skewnormal distribution;

2. Once the shape parameter $\alpha$ is determined, this changes the variance of the B-normal distribution compared to a normal distribution. Adjust the scale parameter $\omega$ until the variance of the B-normal distribution is $\sigma^{2}$ and thus coincides with the corresponding normal distribution;

3. Since the shape and variance have been determined, this in turn changes the expected value of the distribution. To obtain the desired expected value $E(X)$, use the standard formula for the mean of a skew-normal distribution and solve for the location parameter $\xi$. 
This procedure will yield the parameters $\alpha, \omega^{2}$, and $\xi$, and once these have been obtained, the B-normal distribution is parametrically determined. From this distribution, the belief (or confidence) in the different expected values can be determined in the same way as with standard normally distributed information. This is the core of the evaluation step using the B-normal distribution method. In real-life usage of the framework and the tool, a user will iterate repeatedly between the steps and phases within the steps. This paper merely describes the essentials of each step. Next, we try to illuminate the decision process supported by the tool using a real-life decision example from industry that was solved and decided using the DecideIT software tool. The example in the next section illuminates the discriminative power of second-order information since although the resulting alternative values are overlapping, we can quite strongly conclude a clear preference order.

\section{Large-Scale Example}

What sets DecideIT apart is the capacity to enter vague information in a large variety of ways and nevertheless get a solid result as is shown in the following example. The example is slightly simplified to not obscure the central features of the analyses. It is also anonymised but still reflects the essence of the original real-life case. It involves the management of a hydropower plant that experiences sensitive weeks in the late autumn when temperatures start to fall below $0^{\circ} \mathrm{C}$ and the water freezes. At this stage, it is very important to let ice form with a thick enough surface, acting as a coating, to allow water to run free below it. Otherwise, there is a risk of frazil ice jamming the flow, possibly causing turbine failure, and also a risk of having to bypass the water flow, possibly causing flooding downstream and damage to critical infrastructure. However, allowing the ice to form a surface typically requires limiting power production, and this may result in large opportunity costs if the market price of electric power is high, which it typically is when the temperature drops. The decision problem thus involves whether to proceed with the production according to the normal sales plan, based upon certain prices (Alt. 1), or to reduce the runoff according to a predefined setting to allow an ice surface to form (Alt. 2).

Due to the multi-faceted consequences of frazil ice and floods, the firm considered the following seven evaluation criteria:

$$
\begin{aligned}
& \text { Cr. 1: Direct opportunity costs } \\
& \text { Cr. 2: Indirect losses } \\
& \text { Cr. 3: Power station safety } \\
& \text { Cr. 4: Civil utility safety } \\
& \text { Cr. 5: Public safety } \\
& \text { Cr. 6: Local badwill } \\
& \text { Cr. 7: Global badwill }
\end{aligned}
$$

For each criterion, three potential scenarios were defined, and these were modelled as three uncertain consequences, each corresponding to one scenario, with interval probabilities. Scenario 1 corresponded to the most likely scenario with no severe 
consequences, Scenario 3 corresponded to the worst-case scenario, and Scenario 2 was an in-between scenario akin to a previous situation that had occurred approximately ten years prior to the analysis. The probabilities were assessed by an expert panel consisting of on-site hydrologists and operations managers and were intentionally delivered with imprecision, due to a scarcity of historical data and the complexity of the causal effects of an increase in water level. Figure 2 shows a screenshot of the software with the criteria model window, and Figure 3 shows a decision tree for a criterion, with the outcomes for each scenario under the two alternatives. Thus, there was one such tree for each of the seven criteria.

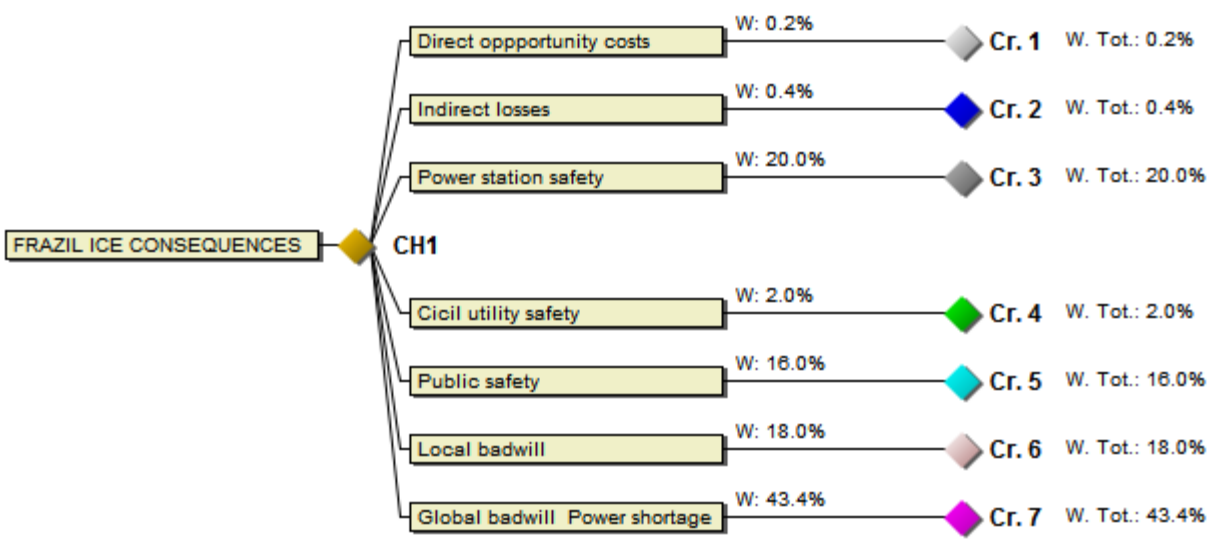

Figure 2 Screenshot of software with window holding the criteria model. Beneath each node, the corresponding scenario decision tree is written out and can be accessed by a mouse click.

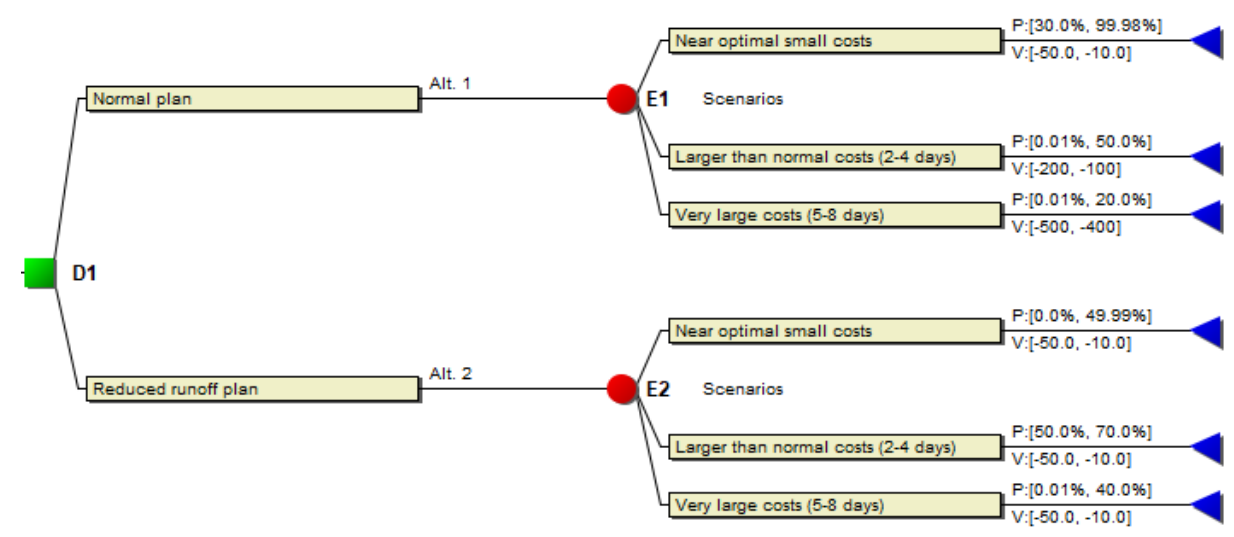

Figure 3 Scenario decision tree for Criterion 1 showing direct opportunity costs, with interval probabilities and interval values for consequences

The values of the consequences were either pointwise or interval estimates of the monetary costs the firm would incur should a particular scenario occur. The criteria weights were calibrated to yield a one-to-one trade-off such that, for example, one Euro 
in direct cost would equal one Euro in cost incurred from securing public safety. To sum up, the overall decision problem is shown in Table 1.

Table 1. Criteria weights, scenario probabilities, and consequence values

\begin{tabular}{|l|l|l|l|l|}
\hline Criterion, weight & Alternative & Scenario 1 & Scenario 2 & Scenario 3 \\
\hline Cr. 1 & Alt. 1 & $p \in[0.3 ; 1]$ & $p \in[0.01 ; 0.5]$ & $p \in[0.01 ; 0.2]$ \\
& & $v \in[-50 ;-10]$ & $v \in[-200 ;-100]$ & $v \in[-500 ;-400]$ \\
\cline { 2 - 5 }$w_{1}=0.002$ & Alt. 2 & $p \in[0 ; 0.5]$ & $p \in[0.5 ; 0.7]$ & $p \in[0 ; 0.4]$ \\
& & $v \in[-50 ;-10]$ & $v \in[-50 ;-10]$ & $v \in[-50 ;-10]$ \\
\hline Cr. 2 & Alt. 1 & $p \in[0.12 ; 0.23]$ & $p \in[0.75 ; 0.85]$ & $p \in[0.02 ; 0.03]$ \\
$w_{2}=0.004$ & & $v \in[-50 ;-10]$ & $v \in[-200 ;-100]$ & $v \in[-400 ;-100]$ \\
\cline { 2 - 5 } & Alt. 2 & $p \in[0.39 ; 0.6]$ & $p \in[0.4 ; 0.6]$ & $p \in[0 ; 0.01]$ \\
& & $v \in[-50 ;-10]$ & $v \in[-200 ;-100]$ & $v \in[-1000 ;-500]$ \\
\hline Cr. 3 & Alt. 1 & $p \in[0.89 ; 1]$ & $p \in[0.01 ; 0.1]$ & $p \in[0 ; 0.01]$ \\
$w_{3}=0.2$ & & $v=0$ & $v=-50$ & $v=-50000$ \\
\cline { 2 - 5 } & Alt. 2 & $p \in[0.97 ; 0.99]$ & $p \in[0.01 ; 0.02]$ & $p \in[0 ; 0.01]$ \\
& & $v=0$ & $v=-50$ & $v=-50000$ \\
\hline Cr. 4 & Alt. 1 & $p \in[0.7 ; 0.9]$ & $p \in[0.1 ; 0.2]$ & $p \in[0.01 ; 0.1]$ \\
$w_{4}=0.02$ & & $v=0$ & $v=-500$ & $v=-5000$ \\
\cline { 2 - 5 } & Alt. 2 & $p \in[0.93 ; 0.98]$ & $p \in[0.01 ; 0.05]$ & $p \in[0.01 ; 0.02]$ \\
& & $v=0$ & $v=-500$ & $v=-5000$ \\
\hline Cr. 5 & Alt. 1 & $p \in[0.97 ; 0.99]$ & $p \in[0.01 ; 0.02]$ & $p \in[0 ; 0.01]$ \\
$w_{5}=0.16$ & $v=0$ & $v=-300$ & $v=-40000$ \\
\cline { 2 - 5 } & Alt. 2 & $p \in[0.99 ; 1]$ & $p \in[0.97 ; 0.99]$ & $p=0$ \\
& & $v=0$ & $v=-300$ & $v=-40000$ \\
\hline Cr. 6 & Alt. 1 & $p \in[0.85 ; 0.99]$ & $p \in[0.01 ; 0.1]$ & $p \in[0 ; 0.05]$ \\
$w_{6}=0.18$ & & $v=0$ & $v=-1000$ & $v=-45000$ \\
\cline { 2 - 5 } & Alt. 2 & $p \in[0.98 ; 1]$ & $p \in[0 ; 0.01]$ & $p \in[0 ; 0.01]$ \\
& & $v=0$ & $v=-1000$ & $v=-45000$ \\
\hline Cr. 7 & Alt. 1 & $p \in[0.89 ; 1]$ & $p \in[0 ; 0.1]$ & $p \in[0 ; 0.01]$ \\
$w_{7}=0.43$ & & $v=0$ & $v=-1500$ & $v=-100000$ \\
\cline { 2 - 5 } & Alt. 2 & $p \in[0.97 ; 1]$ & $p \in[0 ; 0.02]$ & $p \in[0 ; 0.01]$ \\
& & $v=0$ & $v=-1500$ & $v=-100000$ \\
\hline
\end{tabular}

The weighted expected value intervals of the two alternatives then become

$\left[\min \left(E\left(A_{1}\right)\right) ; \max \left(E\left(A_{1}\right)\right)\right]=[-11 ;-1.4]$

$\left[\min \left(E\left(A_{2}\right)\right) ; \max \left(E\left(A_{2}\right)\right)\right]=[-4.8 ;-0.2]$

which clearly overlap. However, when additionally utilising second-order information (see below), we can make use of new analytical means for decision evaluation, foremost support and remaining mass, and here the new software features demonstrate their practical usability.

The support for alternative $A_{i}$ as compared to alternative $A_{j}$ is the joint belief mass where $E\left(A_{i}\right)>E\left(A_{j}\right)$. The remaining mass relies on the concept of contraction. Contraction analysis consists of shrinking the outer feasible boundaries of the expected value for each alternative while measuring $\max \left[E\left(A_{i}\right)-E\left(A_{j}\right)\right]$. The contraction level is indicated as a percentage, where at a $100 \%$ level of contraction, all feasible boundaries have been reduced to points (see Danielson and Ekenberg, 2007). The contraction level 
when $\min \left[E\left(A_{i}\right)-E\left(A_{j}\right)\right]>0$ (or the complementary $\max \left[E\left(A_{i}\right)-E\left(A_{j}\right)\right]<0$ ) is called the intersection level, and the joint belief mass remaining over the contracted orthogonal hull, when the intersection level is reached, is the remaining mass. The more mass that remains after the intersection level, the more confidence we can have in the final outcome of the analysis. See Figure 4 for a so-called robustness graph evaluation showing these results for the current example. In the figure, the upper graph depicts the maximum difference $\max \left[E\left(A_{1}\right)-E\left(A_{2}\right)\right]$ and the bottom depicts $\min \left[E\left(A_{1}\right)-E\left(A_{2}\right)\right]$. These boundary graph lines form a cone that shrinks as the contraction increases, i.e. as the intervals are having more and more of their outer boundaries cut off toward a centre (focal) point. In the figure, it can be seen that as a result of the analysis $98 \%$ of the belief mass lies in the region where $E\left(A_{1}\right)-E\left(A_{2}\right)<0$ which is a very confident result indeed and thus serves as a solid recommendation for a decision-maker. Furthermore, another result of the analysis is that $95 \%$ of the belief mass lies in the region to the right of the intersection with the $x$-axis, i.e. where $\max \left[E\left(A_{1}\right)-E\left(A_{2}\right)\right]<$ 0 (remaining mass). Both of these results together point to a clear-cut result, which would not have been possible to obtain without exploiting second-order information in the decision evaluation.

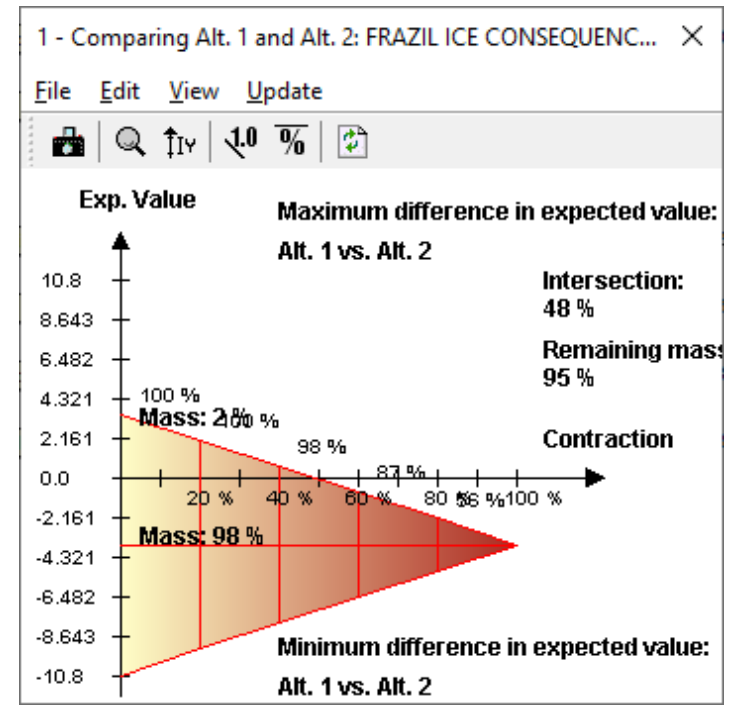

Figure 4 Second-order decision evaluation. Screenshot from DecideIT software version 3.0.

Another evaluation in the DecideIT tool involves stacked bar charts of part-worth values for each criterion in combination with support information. The part-worth value $\varphi_{i l}$ for each alternative $A_{i}$ under Criterion 1 is simply given by $\varphi_{i l}={ }^{c} w_{1} \cdot{ }^{c} V_{i l}$ where ${ }^{c} w_{1}$ and ${ }^{c} V_{i l}$ are the focal point weight for Criterion $l$ and the focal point expected alternative value for alternative $A_{i}$ under Criterion l, respectively. The height of each bar is then the sum $\varphi_{i 1}+\varphi_{i 2}+\ldots+\varphi_{\text {in }}$ for $n$ criteria, which represents the aggregated value of each alternative. However, since this information is imprecise, the stacked bar charts are supplemented with support information, indicating confidence in the resulting ranking. $90 \%$ support is required for the result to be considered a confident result. 


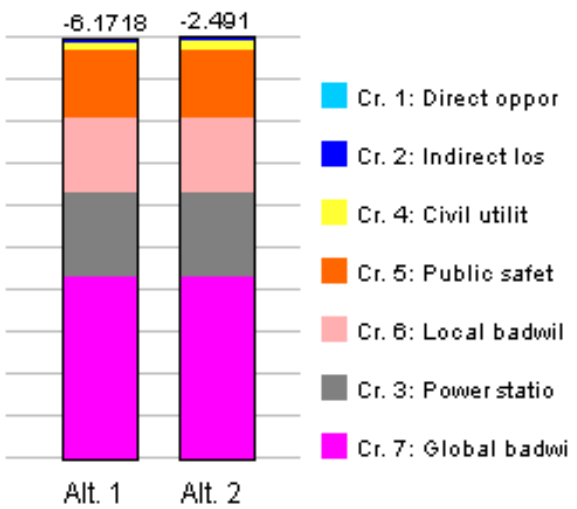

Alt. 1

Alt. 2 >

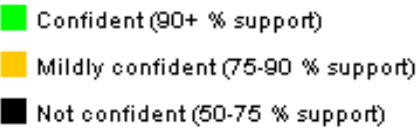

Not confident (50-75 $\%$ support)

Figure 5 Stacked bar chart evaluation showing the value contribution to an alternative from each criterion, together with the results of a support analysis showing that the support is $98 \%$.

In Figure 5, the height of the bars is normalized relative to the highest bar, which in this case is the bar for Alt. 2 which (as was seen in Figure 3) has a significantly higher value in absolute terms. But in the figure, the value differences are relative to the minimum value of the selected main scale corresponding to "Direct cost" which is -500 . Therefore, the bars seem to have similar heights, despite a substantial difference. The overall conclusion of the example is that Alt. 2, reduce the runoff to allow an ice surface to form, was clearly to prefer. This was also the action that was undertaken as a consequence of the decision analysis performed.

\section{Concluding Remarks}

In real-life problems, it is usually impossible to assign precise numerical values to the different components of a decision model, and there is hence a need for representation and evaluation mechanisms that can effectively handle information incompleteness. Higher-order analyses such as belief mass can add both information and transparency, thus enabling a much more discriminative analysis than using intervals alone. We describe a higher-order framework realised by a software tool, based on an evaluation method using a belief mass interpretation of the data involved. We discuss a model and 
its implementation where second-order information is used for analysing both decision trees and a multi-criteria models, and as a demonstration, we apply it to an actual reallife decision problem from industry (power generation) to illustrate our new software features by demonstrating how second-order effects affect the resulting distribution over the expected values. The framework presented in this paper is put to use for solving decision situations using the tool that packages the framework and makes it available to real-life decision-makers with limited mathematical and decision theoretical knowledge, but an understanding of the decision context. Future work includes the design and development of efficient elicitation methods taking advantage of belief mass interpretations, and applications of the framework and accompanied software tools for analysing complex decision problems in business and public policy.

Acknowledgements. This research was supported by the EU-project Co-Inform (CoCreating Misinformation-Resilient Societies H2020-SC6-CO-CREATION-2017) and strategic grants from the Swedish government within ICT - The Next Generation. This paper partially builds on an earlier short paper presented at the conference ISIPTA'19 in Gent, Belgium.

\section{References}

B.S., Ahn, Compatible weighting method with rank order centroid: Maximum entropy ordered weighted averaging approach, European Journal of Operational Research, pp.552-559, 2011.

B.S. Ahn, and K.S. Park, Comparing methods for multiattribute decision making with ordinal weights, Computers \& Operations Research 35 (5), 1660-1670, 2008.

A. Arbel, and L.G. Vargas, Preference simulation and preference programming: robustness issues in priority derivation, European Journal of Operational Research 69, 200-209, 1993.

F.H. Barron, Selecting a Best Multiattribute Alternative with Partial Information About Attribute Weights. Acta Psychologica 80(1-3), pp. 91-103, 1992.

F. Barron and B. Barrett, The Efficacy of SMARTER: Simple Multi-Attribute Rating Technique Extended to Ranking. Acta Psychologica 93(1-3), 23-36, 1996a.

F. Barron and B. Barrett, Decision Quality Using Ranked Attribute Weights. Management Science 42(11), 1515-1523, 1996b.

O. Caster, N. Norén, L. Ekenberg and R. Edwards, Quantitative Benefit-Risk Assessment using only Qualitative Information on Utilities, Medical Decision Making, 32(6), pp. E1-E15, 2012.

M. Danielson and L. Ekenberg, Computing Upper and Lower Bounds in Interval Decision Trees, European Journal of Operational Research 181 (2007) 808-816.

M. Danielson, L. Ekenberg, and A. Larsson, Belief Distribution in Decision Trees, International Journal of Approximate Reasoning, 46(2) (2007) 387-407.

M. Danielson, L. Ekenberg, A. Larsson, and D. Sundgren, Second-Order Risk Constraints in Decision Analysis. Axioms 3 (2014) 31-45.

D. Dubois, Representation, Propagation, and Decision Issues in Risk Analysis Under Incomplete Probabilistic Information, Risk Analysis 30(3) (2010) 361-368.

P. Dutta, Human Health Risk Assessment Under Uncertain Environment and Its SWOT Analysis, The Open Public Health Journal 11 (2018) 72-92. 
L. Ekenberg and J. Thorbiörnson, Second-Order Decision Analysis, International Journal of Uncertainty, Fuzziness and Knowledge-Based Systems, 9(1) (2001) 13-38.

L. Ekenberg, M. Danielson, and J. Thorbiörnson, Multiplicative Properties in Decision Trees, International Journal of Uncertainty, Fuzziness, and Knowledge-Based Systems 14(3) (2006) 293-316.

L. Ekenberg, M. Danielson, A. Larsson, and D. Sundgren, Second-Order Risk Constraints in Decision Analysis, Axioms, 3 (2014) 31-45.

T. Fasth, S. Bohman, A. Larsson, L. Ekenberg and M. Danielson, Portfolio Decision Analysis for Evaluating Stakeholder Conflicts in Land Use Planning, to appear in Group Decision and Negotiation.

R. Fuller and P. Majlender, An analytic approach for obtaining maximal entropy OWA operator weights. Fuzzy Sets and Systems 124, pp. 53-57, 2001.

R. Fuller and P. Majlender, On obtaining minimal variability OWA operator weights. Fuzzy Sets and Systems 136, pp. 203-215, 2003.

D. Golenko-Ginzburg, Controlled alternative activity networks in project management, European Journal of Operational Research 37 (1998) 336-346.

K. Hansson, A. Larsson, M. Danielson and L. Ekenberg, Coping with Complex Environmental and Societal Risk Management Decisions: An Integrated Multi-Criteria Framework, Sustainability, 3(9), pp. 1357-1380, 2011.

F. Herrera and E. Herrera-Viedma. Linguistic decision analysis: steps for solving decision problems under linguistic information. Fuzzy Sets and Systems 115 (1), (2000) 67-82.

C. Jansen, G. Schollmeyer, and T. Augustin, Concepts for decision making under severe uncertainty with partial ordinal and partial cardinal preferences. International Journal of Approximate Reasoning 98, (2018) 112-131.

E.T. Jaynes, Prior Probabilities, IEEE Trans. on Systems Science and Cybernetics, SSC-4, 227, 1968.

K. Katsikopoulos and B. Fasolo, New Tools for Decision Analysis. IEEE Transactions on Systems, Man, and Cybernetics - Part A: Systems and Humans 36(5), 960-967, 2006.

M.G. Kendall and A. Stuart, The Advanced Theory of Statistics, Volume 1: Distribution Theory, $3^{\text {rd }}$ edition, Griffin, 1969.

F. Kivunike, L. Ekenberg, M. Danielson and F. Tusubira, Using a Structured Approach to Evaluate ICT4D: Healthcare Delivery in Uganda, The Electronic Journal of Information Systems in Developing Countries, Vol. 66(8), pp. 1-16, 2015.

N. Komendantova, L. Ekenberg, L. Marashdeh, A. Al-Salaymeh, M. Danielson and J. Linnerooth-Bayer, Are Energy Security Concerns Dominating Environmental Concerns? Evidence from Stakeholder Participation Processes on Energy Transition in Jordan, Climate, 2018.

S. Kotz and J.R. van Dorp, Beyond Beta, Other Continuous Families of Distributions with Bounded Support and Applications, World Scientific Press, Singapore, 2004.

A. Larsson, L. Ekenberg, M. Danielson, Decision Evaluation of Response Strategies in Emergency Management using Imprecise Assessments, Journal of Homeland Security and Emergency Management, Vol. 7:1, pp. 1547-7355, 2010.

A. Larsson, A. Kuznetsova, O. Caster, and L. Ekenberg, Implementing Second-Order Decision Analysis: Concepts, Algorithms, and Tool, Advances in Decision Sciences, 2014.

M. Loeve, Probability Theory. Graduate Texts in Mathematics, Volume 45, $4^{\text {th }}$ ed., SpringerVerlag, 1977. 
A. Mihai, A. Marincea, and L. Ekenberg, A MCDM Analysis of the Roşia Montană Gold Mining Project, Sustainability Vol. 2015(7), pp. 7261-7288, doi:10.3390/su7067261, 2015.

Preference AB, The DecideIT Decision Tool, available at www.preference.nu, 2019. Available for free for academic and other non-commercial use.

J. Rohmer and C. Baudrit, The use of the possibility theory to investigate the epistemic uncertainties within scenario-based earthquake risk assessments, Natural Hazards 56(3) (2010) 613-632.

M. Riabacke, M. Danielson, and L. Ekenberg, State-of-the-Art in Prescriptive Weight Elicitation, Advances in Decision Sciences, vol. 2012, Article ID 276584, 24 pages, 2012.

A.F. Shapiro and M.C. Koissi, Risk Assessment Applications of Fuzzy Logic. Casualty Actuarial Society, Canadian Institute of Actuaries, Society of Actuaries, 2015.

T.J. Stewart, Use of Piecewise Linear Value Functions in Interactive Multicriteria Decision Support: A Monte Carlo Study, Management Science 39(11), 1369-1381, 1993.

W. Stillwell, D. Seaver, and W. Edwards, A Comparison of Weight Approximation Techniques in Multiattribute Utility Decision Making. Organizational Behavior and Human Performance 28(1), pp. 62-77, 1981.

D. Sundgren, M. Danielson, and L. Ekenberg, Warp Effects on Calculating Interval Probabilities, International Journal of Approximate Reasoning, 50(9) (2009) 1360-1368.

T. Tervonen and R. Lahdelma, Implementing Stochastic Multi-criteria Acceptability Analysis, European Journal of Operational Research, 178(2) (2007) 500-513.

Yager, R.R., On ordered weighted averaging aggregation operators in multi-criteria decision making, IEEE Transactions on Systems, Man and Cybernetics, B 18, pp. 183-190, 1988. 